\title{
CORRIGENDUM
}

\section{Comparison of large-insert, small-insert and pyrosequencing libraries for metagenomic analysis}

\author{
Thomas Danhorn, Curtis R Young and Edward F DeLong
}

The ISME Journal (2012) 6, 2152; doi:10.1038/ismej.2012.52

Correction to: The ISME Journal (2012) doi: 10.1038/ ismej.2012.35; published online 26 April 2012

Since the publication of this article, the authors have noticed a reference omission (Ghai et al., 2010) that should have been cited in the article.

By adding the citation, the following sentence has been deleted from page 2:

The above studies, however, were each based on a single sample and a relatively small number of sequenced clones, complicating a comprehensive comparison.
And replaced by:

Subsequently, Ghai et al. (2010) reported an apparent GC bias and under representation of Pelagibacter and Prochlorococcus clones in picoplankton fosmid libraries from a Mediterranean deep-chlorophyll maximum layer.

The above citation, along with the reference for Ghai et al. (2010) have now been added to the paper, and the correct article appears in this issue. The html and online pdf versions have also been rectified, and now carry the correct paper.

The authors would like to apologise for any inconvenience this may have caused. 